\title{
Computer Assisted Counter System for Larvae and Juvenile Fish in Malaysian Fishing Hatcheries by Machine Learning Approach
}

\author{
Valliappan Raman ${ }^{1}$, Sundresan Perumal2 ${ }^{2}$, Sujata Navaratnam ${ }^{3}$, Siti Fazilah ${ }^{3}$ \\ ${ }^{1}$ Multimedia Research Group, School of Computer Science, Universiti Sains Malaysia, Malaysia. \\ 2 Faculty of Science and Technology, Universiti Sains Islamic Malaysia, Malaysia. \\ ${ }^{3}$ Department of Computing, KDU University College, Malaysia. \\ * Corresponding author. Tel.: 0060164404636; email: Sundresan.p@usim.edu.my \\ Manuscript submitted April 4, 2015; accepted November 20, 2015. \\ doi: 10.17706/jcp.11.5.423-431
}

\begin{abstract}
The increased in number and size of larvae and juvenile growth are estimated based on manual approach in fishing hatcheries. There is a high demand for computer assisted software solution for aquaculture research in early detection and recognition of fish population. There exist several companies who have introduced fish detection technologies into the market. Although able to count the number of larvae with a high accuracy rate, the fish counter software's may encounter difficulties when detecting smaller larvae's and ants in very early stage of birth period. The main aim of the paper is to propose a framework using machine learning techniques that can be of low cost and efficient system for fish counting and growth study. The expected final result will be a complete preliminary prototype with basic camera setup, focus on larval fish. medium term, improve camera setup and quality; focus on larval and juvenile fish. For the Long term, full fish growth tracking and data mining is implemented. The proposed research in this paper will assist the Malaysian fisheries department to have accuracy on detecting the larvae, juvenile and ants in the hatcheries.
\end{abstract}

Key words: Aquatic, enhancement, segmentation and counting.

\section{Introduction}

The early life history of fish is often considered critical for recruitment to the adult population. Thus, understanding how larval fish population's distribution varies through time and among habitats is important for answering questions related to both applied and theoretical ecology in marine research. The global demand for aquaculture software, supplies and equipment is predicted to exceed $\$ 80$ billion by 2016 due to increasing demand for fish in the market. Commercialization of farming across the world and increasing intensities of production will necessitate the use of commercial grade feeds, chemicals and biological enhancements as well as more mechanization on fish farms. Therefore, demand for fish is expected to continually outpace growth in other global food sources such as livestock and agriculture. The Asia Pacific region is projected to account for over 80 percent of global demand for aquaculture software supplies and equipment by 2016. This is in direct correlation to the region's global dominance in aquaculture production. Several factors contribute to the huge aquaculture production turnover from Asia: a long coastline; tropical and temperate weather suitable for rearing aquatic species; low labor costs in a hitherto labor intensive industry; strong local demand as well as export competitiveness; integrated 
farming practices enabling multiple crops, better land and resource utilization; and a growing manufacturing base for supplies and inputs. Due to the increasing exploration on the fishery industry, new method had to be explored in order to support it and to keep it sustainable. Machine learning approach might give solution for supporting the fishery industry. For instance, it can help to detect, recognize, and analyze the fish by using image processing techniques and it will be faster, easier and convenient than the manual way. But there are several problems in detecting and recognizing the fish in the image such as the target object is obscured due to the presence of the other object which can interfere with recognition process and any other noise. It makes the system difficult to identify the feature of the fish stages.

In fishing hatcheries, when the reproduction system of fish breeding starts from egg to spawning stage. Each fish species has a unique reproductive strategy and favors certain habitats for spawning and for early development of their newly hatched young. Fish life cycles vary among species. In general, however, fish progress through the following life cycle stages: 1) Eggs: Fertilized eggs develop into fish. Most eggs do not survive to maturity even under the best conditions. Threats to eggs include changes in water temperature and oxygen levels, flooding or sedimentation, predators and disease. 2) larval: Larval fish live off a yolk sac attached to their bodies. When the yolk sac is fully absorbed, the young fish are called fry. 3) Fry: Fry are ready to start eating on their own. Fry undergo several more developmental stages, which vary by species, as they mature into adults. 4) Juvenile: The time fish spend developing from fry into reproductively mature adults varies among species. Most fish do not survive to become adults. Threats to survival include fluctuations in water temperature, changes in oxygen levels, competition for habitat and predators. 5) Adult: When fish are able to reproduce, they are considered adults. 6) Spawning: Female fish release eggs into the water and male fish fertilize eggs by releasing milt. Not all eggs are fertilized. Some fish spawn each year after reaching maturity; others spawn at intervals while others spawn only once and then die.

Our research involves in counting the larvae and juvenile in second and fourth stage of the cycle which is important for aquatic researchers to monitor the growth of fish by categorized into alive, partially alive, and not alive. However, the counting process is time consuming and can be subjected to human error. In this paper, we explore how this counting can be automated using image processing approaches. Early studies have shown that some possible ways of fish counting. However, they are designed for bodies of water with different varieties of fish so they include some form of fish species detection algorithm so as to be able to count the number for each species. In addition, fish tracking algorithms are also used in the counting of fish. Thus, their suggested methods are computationally intensive and will increase costs and slow down the counting speed.

The key idea behind our proposed method is the use of camera to mark the position of the fish breeding in the hatcheries, and then analyzing the area of each image to decide how many larvae's and juvenile are captured in each image. This in turns yields the number of fish in a multiple images. Individual images from various positions are processed separately, and the number of fish computed from each image is then added up and divided by the total number of images from multiple locations to obtain an average count.

The paper is organized as follows: Section 2 explain the research challenges for counting the early stages of larvae in the hatcheries. Section 3 describes the research objective for the research carried out in the paper. Existing works and commercial software products for counting the fish in market is explained in Section 4. Machine learning approaches are explained in detail at Section 5. The initial results of the proposed method are shown in Section 6. Finally conclusion is made in Section 7.

\section{Research Challenges}

Detection and recognizing an object is very difficult and takes a long times especially for the live object. In fisheries industries, species of the fish must be recognizing for calculating their population. Currently, 
manual approach is done by an official of fisheries department one by one for calculating and measuring fish length. One of the fundamental challenging problems in computer vision is detecting object inside an image or video frames. Therefore monitoring larval fish production in freshwater assesses the overall effectiveness of habitat restoration in lakes and rivers across the nation have challenges as follows: 1) Aquaculture research, to calculate the livestock of larvae, manually performed by human. 2) Count accuracy rate was very poorly determined. 3) High error margin with less performance result.4) Larva growth information's are not properly maintained and integrated.

\section{Research Objectives}

According to the Department of Fisheries Malaysia statistics, the market value of marine fishes have increased significantly from the year 2000 (RM 4 billion) to 2009 (RM 6 billion) with a growth rate of around 50\% [1]. Furthermore, based on the Tenth Malaysia Plan, high value agriculture activities including aquaculture shall be focused upon [2].From this plan, the government plans to introduce modern technology to improve production processes as well as intensify research and development, in hopes to create new high-value added products. These points give reason to the creation of an aquaculture software solution. The research aims to count and measure the total number of larvae fish through image processing techniques. This provides users with accurate data concerning larvae production which in turn leads to better planning and decision making. Additionally, the software based larvae detection system provides improved reliability, efficiency and accuracy when estimating the total number of fish. The main research objectives leads for the development of larvae counter system are as follows:

- Develop a machine learning algorithm to Count and Measure the number of larvae fish and to calculate livestock value, food requirements and cost.

- Provide the user accurate information about the production or catch process and so facilitate planning and decision-making.

- Achieve higher accuracy rate with good performance and reliability.

- Deliver larvae information report on reproduction and adult stages.

The proposed research solution, the fish counter intends to address several existing issues regarding larval and juvenile fish counting.

\section{Existing Works}

There are few existing research works carried out in the past for counting the fishes based on the legth and size of measurement in adult stage not in young stage. The research carried out in the past are by using image processing method. In 2006, D. J. White [3] developed a machine system for sorting some fish species by employing modern hardware and programming techniques in real time. The system is called as Catch Meter, which includes conveyor, light box and feeder. The mechanical systems were controlled by a PLC interfaced to the main computer and software via an Ethernet link. Fish moved along the conveyor belt at $1.5 \mathrm{~m} / \mathrm{s}$ and was analyzed by the computer vision system. Fish detection also was done using camera video like Williams' experiment [4]. Researcher constructed a prototype system for detecting and delineating fish shapes in video footage obtained from a local aquaculture facility. Another method is to use edge detection to identify the fish [5]. The canny edge detector is used because it provides the most number of true edges [6]. To eliminate the edges of the container, the image is filled with redundant black data starting from the sides of the image outwards. This set up an additional rectangular edge denoting the original outer border of the image. Morphological closing is done next to join the edges that were not terminated properly and to fill in the areas. Blobs are thus created from the edges. This step is also necessary to connect the round edges of the container to the original outer border of the image so they can all be eliminated by eliminating 
blobs which has a major axis much longer than the length of five fish. The final results are obtained after eliminating noise and background objects. This results in inaccuracies when we use the area to count the number of fish in a blob. These methods are not promising to produce accuracy and reliability in counting the number of fishes from hatcheries.

There are few commercial products available in the market to count the fish from the different type of environment. Several competing companies have introduced fish counting and monitoring solutions that enable the detection of fishes through specialized equipment. These tools range from light counters which detect fish through underwater lights, to sound waves which count the number of fish. Although capable of accurately counting fish, these options fail to detect fish under certain measurements (e.g. below $5 \mathrm{~cm}$ ). Furthermore, the cost of acquiring such equipment may be high (e.g. around \$75,000 and above). Vaki, an Icelandic company, allows for fish counting by a light valve. When a fish passes their counter, light is blocked, therefore indicating that a fish is present. Although able to count fish with a high accuracy rate, the Vaki fish counter may encounter difficulties when detecting smaller fish. Furthermore, it may function incorrectly when used in turbid water [7]. The company, Sound Metrics, provides Dual frequency identification sonar (DIDSON) which detects fish through sound waves. Underwater pictures are produced which are later processed with fish recognition software. Although high quality images can be produced, the DIDSON module also faces issues when counting small (below $5 \mathrm{~cm}$ ) fish. Additionally, the cost for each DIDSON unit is expensive with prices from $\$ 75,000$ and above [8]. AKVA Group with their Fishtalk software enables users to store aquaculture data as well as create reports. Moreover, the software is not species dependent meaning that it can be compatible with numerous fishes. Although this is so, the Fishtalk software does not specifically support monitoring larval fish, and therefore may omit collecting certain important information [9]. The current work differs from these existing products in terms of cost as well as hardware and software usage. Though other solutions offer specialized tools, they must be directly purchased and may incur a high price. Additionally, most of these tools are not able to accurately estimate the larval fish population due to their reduced size. In contrast, the proposed prototype can function with any normal camera that provides sufficient picture quality, consequently reducing equipment costs. Furthermore, custom larvae detection software enables the counting of small larval fish which may remain unrecognized by other solutions [10] [11].

Furthermore, research has been undertaken to improve support towards fisheries which leads to better aquaculture development. The main benefits from the outcome of this research include fish farmers, hatcheries or marine researchers who require low cost solutions in improving their fish production or aquaculture research. Since the outcome of this research is a prototype which only requires a camera and recognition software by using machine learning approaches, it is fit for any research experts or industry wanting to detect and study larval and juvenile fishes.

\section{Methodology}

Larvae and Juvenile detection and recognition system is an image processing technology used to detect the young larves and juvenile in an image and recognize the number of larves and juvenile present in an image. Therefore the main aim of this paper is to segment the images into separate single image and it is counted. Segmentation is used for detecting the edges in an image. The purpose of segmenting an image into a set of disjoint areas/segments is to simplify the image. The image may be further simplified by assembled the small areas into larger objects by building a symbolic scale space over the segments. The segmentation in this image is entirely based on the gradient transformation. Segmentation and the choice of segmentation algorithm cannot be proven optimal to the task of recognizing objects in any strict sense. The gradient transform has proven itself useful in many contexts, but the choice is heuristic and can only be 
justified by experience. In the case of recognition of objects such as young larvae and juvenile in images, the object must in some sense be different from its surroundings. Therefore the methodology is categorized in four stages: Image Acquisition, Image Enhancement, Segmentation and Classification, in final classification are performed with counting the number of young larvae and juvenile fishes in the captured image. Below Fig. 1 illustrates the working methodology of machine learning approach.

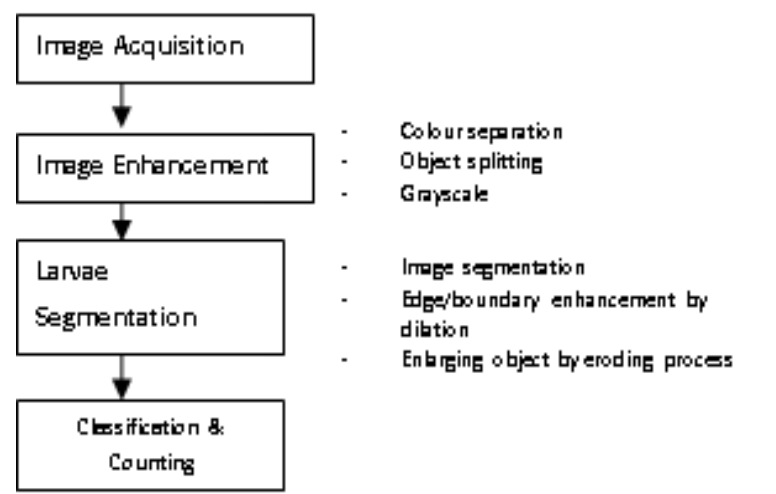

Fig. 1. Illustrates the methodology of machine learning approach.

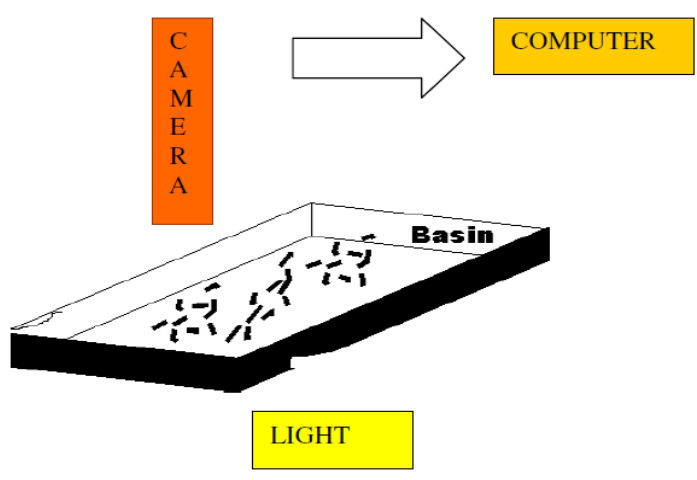

Fig. 2. Illustrates the image acquisition of capturing the larvae image from hatcheries.

\subsection{Image Acquisition}

Images were captured via camera mounted on the aquatic basin (See Fig. 2). Once the camera captures the larvae, it will be transferred to the video software for processing the image. The captured image is converted into grayscale image by color transformation method.

\subsection{Image Enhancement by Sobel Operator}

Larvae and Juvenile can be easily detected in an image if the larvae and Juvenile have sufficient contrast from the background. In order to improve contrast of larvae edges and separate from ants, Sobel operator is applied in the square of the image, which creates a binary mask using a user-specified threshold value. Multiple young larvae are present in the square of image, so that the larvae can be seen in its entirety. The object to be segmented differs greatly in contrast from the background image. Changes in contrast can be detected by operators that calculate the gradient of an image.

\subsection{Segmentation by ROI Hunting and Edge Detection}

First step in the segmentation is to identify the region of interest. Each image containing a square field was processed in order to establish a region of interest. The captured image is processed using a simple algorithm that is able to detect the four vertices of the square field. Horizontal coordinates of these vertices are estimated by observing local minima on a profile function which is obtained with the sum of pixel values over each column of the image. Vertical coordinates are obtained similarly but with a profile function considering the sum over each row of the image.

Second step is to pre-segment the edges in the image. The binary gradient mask shows lines of high contrast in the image. These lines do not quite delineate the outline of the object of interest. Compared to the original image, gaps can be viewed in the lines surrounding the object in the gradient mask. These linear gaps will disappear if the Sobel image is dilated using linear structuring elements. Therefore the gaps are filled. Nest stage where the binary gradient masks is dilated using the vertical structuring element followed by the horizontal structuring element. The dilated gradient mask shows the outline of the larvae cell and ant quite nicely, but there are still holes in the interior of the cell, therefore the gaps are filled in uniform 
format.

The larvae and juvenile cell of interest and ant has been successfully segmented, but it is not the only object that has been found. Any objects that are connected to the border of the image need to be removed. The connectivity was set to 4 to remove diagonal connections, so that objects are separated into individuals and it is detected in order to make the segmented object look natural; smooth the object by eroding the image twice with a diamond structuring element, so that each and every larvae cells and ants are detected and it is easy to count the number of larvae's and ant in image. Therefore while overlapping or contradiction appears in an image, it is detected by boundary detection algorithm. Therefore if there are multiple larvae are overlapped, then considered two nodes and by that the two nodes are replaced by one, their mutual edge is ignored, and the neighbors of the new node is the union of the two original nodes individual neighboring sets. Instead of contracting the two nodes imposed by a connected operation, a new node can be introduced, and the two nodes can both be linked to the new node by edges. Then the full set of nodes (both original and new ones) and the set of edges alone constitute a tree. So that overlapped nodes are separated and it counted easily into one full object in an image.

\subsection{Classification and Counting Method}

Larvae and Juvenile in the image were classified depending on whether they had contact with each other. It is identify, whether the larvae were entirely inside the region of interest or not from the image. Two processes were undergone in the stage; those larvae that were not connected in any way with the border edges and those that were connected in some way. The process of classification is based on neural network approach. The method is divided into two main components: firstly, the recognizing system that uses neural network, and secondly the scanning method that examines each location in the image at several scales, looking for locations that might contain a larvae and juvenile fish. The neural network that is used in this system is multilayer perceptron. The architecture used is based on the work that done by previous work [8]. Multilayer perceptron neural network is one of the conventional feed-forward artificial neural networks. The multilayer perceptron used has 400 input units, which receive input from $20 \times 20$ grayscale pixel from window scanner. The feature used for this work is the edges of the larvae. This is used for the first experiment purpose for this work. When the larvae and juvenile are detected, usually the object would be detected for several times. This makes the detection result are overlapped each other. The object detected should be in one detection area. Therefore the detected objects should be merged. The object candidates are merge by the considering the number of overlapping detection in the method.

Counting of Larvae- Each object is categorized as a single larva or juvenile or a multiple larvae that are connected with each other in the image. Pixel counting was used to calculate each object's area. System processes an image in rows and columns, a pixel is at the intersection of a line and column and the system stores a value for each pixel. Therefore 0 is for no larvae present (black) and 255 for larvae present (white). While determining the area of a larva, then it is needed to calculate the number of larvae/juvenile contained in all images. It is assumed that the area of different objects contained in images can either be of a single larva or of a multiple number of the area of a single larva. While calculating the larvae, histogram is calculated. The histogram shows a periodic behavior that is caused by the fact that multiples of the area of a single larva will be the main objects present in all images. The period in this histogram is considered as a valid interpretation of the area of one larva. Second stage is to apply sobel operator to enhance the contrast from background to detect the larvae. ROI is calculated and boundary detection algorithm is applied to segment and count the number of larvae's/juvenile through connected regions.

\section{Initial Experimental Results}

A testing conducted to give a view of its performance in detecting young larvae and juvenile fish [12]. A 
preliminary experiment was carried out to determine the accuracy rate of the method. Images were captured via camera mounted on an aquatic basin. Once the camera captures the larvae image, it will be transferred to the counter system for image processing. The container acts as the background while the fish are the foreground objects to be extracted. Therefore the container must be bright in color to provide an acceptable contrast to the fish. A video camera records images of the fish for a predefined amount of time. The captured image is then converted into a grayscale image by color transformation methods, enhancement method, segmentation and classification. The data used in this testing divided into two data which are the training data set for training, and the testing data set for the testing. The neural network architecture used in this work is multi-layer perceptron. The system will be given 400 input unit of $20 \times 20$ pixel grayscale image of the sub-image. The output value from the unit will be around -1 and 1 . In the training data set, the value 1 is defined as the larvae edges, while -1 is defined as the non larvae. The result shows that the counter gives a number of false positive and false negative. Some area was detected and somewhere not detected, which need to further developed for accuracy. Currently accuracy achieved in detecting larvae is $82 \%$ and juvenile is $87 \%$.

A simple setup for practical implementation is performed as explained above, Larvae and Juvenile required to be counted flow from a smaller container. These images of larvae and juvenile are processed to produce the count of the fish. The first set of experiment is implemented in MATLAB. The results of the machine learning approach in detecting and counting the larvae and juvenile is shown below from Fig. 3 to Fig. 6.

\subsection{Original Image and GUI Interface}
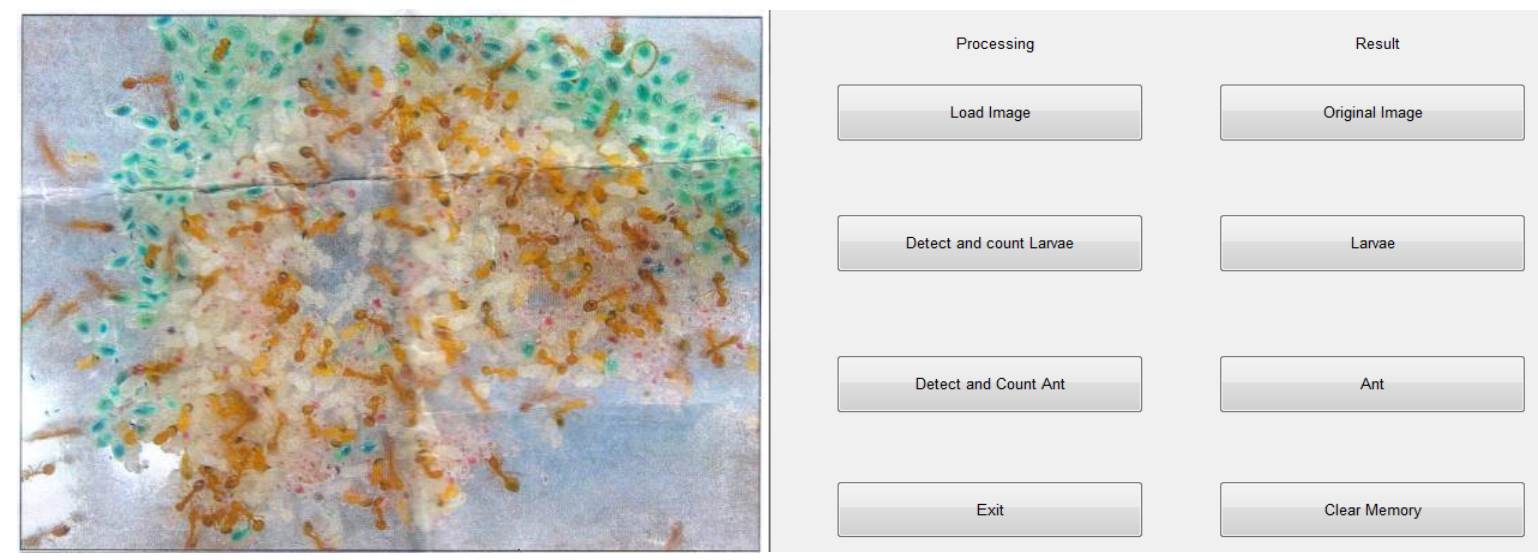

Fig. 3. Illustrates the original image and GUI of the prototype.

\subsection{Image Enhancement}
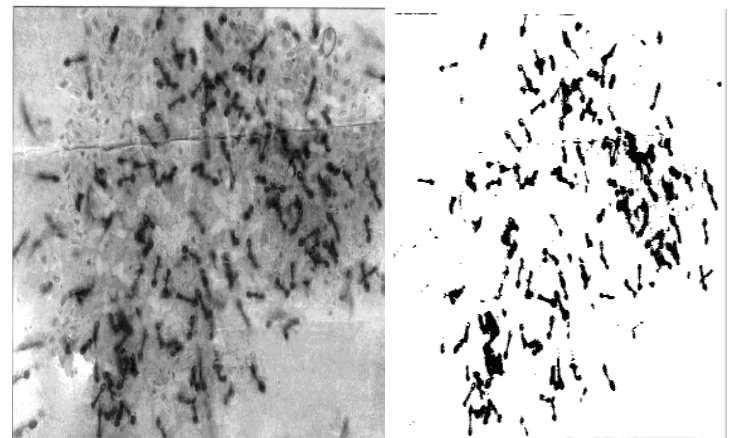

Fig. 4. Illustrates the noise elimination and contrast enhancement by preprocessing stage. 


\subsection{Segmentation}

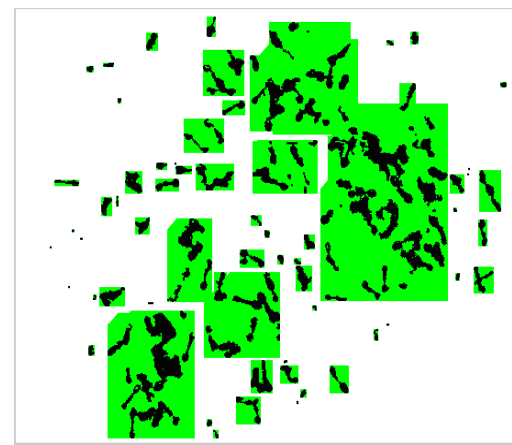

Fig. 5. Illustrates the segmentation by edge detection method.

\subsection{Classification and Counting}

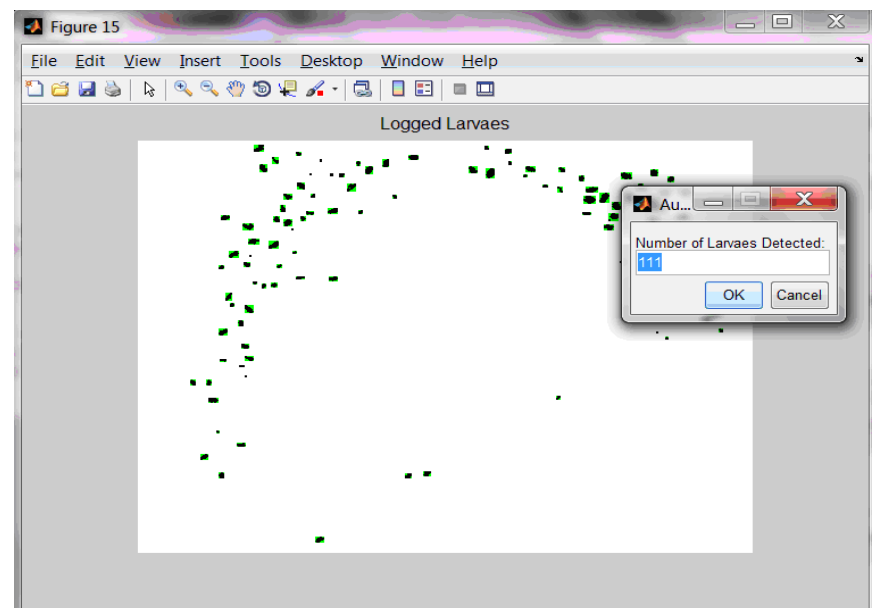

Fig. 6. Illustrates the classification by neural network approach and counting method.

\section{Conclusion}

This research presents one of the object detection techniques for detecting young larvae and juvenile fishes from hatcheries. This work is aimed to study the implementation of machine learning technique in detecting the larvae/juvenile object in a digital image. The result shows that the system is affected by the training data and the fish image that obscured and has a lot of noise. In future work, certain measures can be taken to improve the accuracy of classification and counting the missed larvae's and juvenile fish.

\section{Acknowledgment}

We would like to thank the research centre, KDU university college, Malaysia for funding the project under internal grant scheme for developing the prototype. Would like thank for the facilities and support provided by faculty of computing, KDU for the project.

\section{References}

[1] Official Portal: Department of Fisheries Malaysia. Department of Fisheries Malaysia. From http://www.dof.gov.my/59

[2] Belongie, S., Malik, J., \& Puzicha, J. (2002). Shape matching and object recognition using shape contexts. IEEE Trans. Pattern Anal. Mach. Intell., 24(4).

[3] White, D. J., Svellingen, C., \& Strachan, N. J. C. (2006). Automated measurement of species and length of 
fish by computer vision. Fisheries Research, 80, 203-210.

[4] Williams, R. N., Lambert, T. J., Kelsall A. F., \& Pauly, T. (2006). Detecting marine animals in underwater video: Let's start with salmon. Proceedings of the Twelfth Americas Conference on Information Systems, Acapulco, Mexico.

[5] Boles, W. W., Geva, S., \& Busch, A. (1999). An image processing approach for estimating the number of live prawn larvae in water. Signal Processing and Its Applications, Brisbane, Australia.

[6] Lee, D. J., Schoenberger, R., Shiozawa, D., Xu, X. Q., \& Zhan, P. C. (2004). Contour matching for a fish recognition and migration monitoring system. Proceedings of SPIE Optics East, Two and Three-Dimensional Vision Systems for Inspection, Control, and Metrology II.

[7] Broersen, J. (2009). Towards a Detection and Recognition System for Freshwater Fish (MSc Report).

[8] Pontuala, H. de, Roberta, R., \& Minerb, B. P. (1998). Study of bivalve larval growth using image processing. Journal of Aquaculture Engineering, 17(2).

[9] Johnson, I., Harman, M., Forrow, D., \& Norris, M. (2001). An assessment of the feasibility of using image analysis in the oyster embryo-larval development test. PubMed, Environ Toxicol, 16(1), 68-77.

[10] Borenstein, E., \& Ullman, S. Class-specific, top-down segmentation. ECCV, 2.

[11] Felzenszwalb, P. F., \& Huttenlocher, D. P. (2005). Pictorial structures for object recognition. International Journal of Computer Vision, 61(1).

[12] Valliappan, R., Brian, L., \& Patrick, T. (2011). First prototype of aquatic ToolKit: Towards low-cost intelligent larval fish counting in hatcheries. Proceedings of 9th International Conference on Pervasive Intelligence and Computing (pp. 193-195), Sydney, Australia.

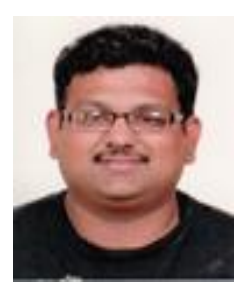

Valliappan Raman is a research fellow at Multimedia Research Group Lab, Universiti Sains Malaysia, Penang, Malaysia. He have worked as a team member to acquire many external research grants and published papers in impact factor journals. His research interests are in medical imaging, data mining and health informatics.

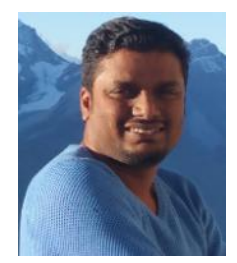

Sundresan Perumal is a senior lecturer in Universiti Sains Islamic Malaysia. He acquired various external research grants and published papers in impact factor journals. His research interests are in cyber security, medical imaging, data mining and network forensics.

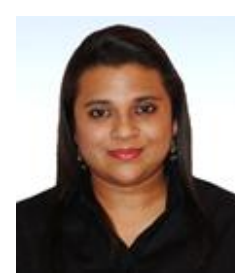

Sujata Navaratnam is a senior lecturer at KDU University College, Malaysia. Her research interests are in image processing, security, ICT in education and software engineering.

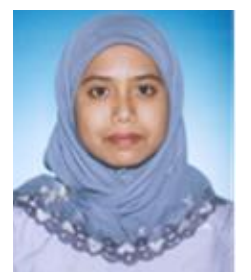

Siti Fazilah Shamsudin is senior lecturer at KDU University College, Malaysia. Her research interests are in software engineering, human computer interaction and computer science education. 